\title{
The locus of stimulus probability effect in choice reaction time
}

\author{
AMOS SPECTOR and RONALD D. LYONS \\ Oakland University, Rochester, Michigan 48063
}

\begin{abstract}
A choice reaction time experiment was conducted in which subjects were to respond with one response to either of two stimuli, S1 and S2, and with another response to a third stimulus, S3. The stimuli in the low-compatibility condition were three colors and the responses were letter names. In a high-compatibility condition, S1 and S2 were upper- and lowercases of the same letter and S3 was another letter. The responses were the letter names. The relative frequency of S1 and S2 was manipulated. The results showed a Compatibility by Relative Frequency interaction, with relative frequency affecting reaction time only under low compatibility. It was argued that the stimulus relative frequency affects the response selection stage of information processing.
\end{abstract}

Using a 1 to 1 stimulus-response (S-R) mapping in a choice reaction time (CRT) task typically shows reaction time to increase with a decrease in the relative frequency of the stimulus. This fact has been shown to hold, at least when S-R compatibility is not particularly high. The increase in reaction time (RT) as a function of decrease in relative frequency is obtained if relative frequency is varied by manipulating the number of equally likely alternatives (Hick, 1952). It is also found when the probability of occurrence of particular stimuli is manipulated within a set of $\mathrm{n}$ possible alternatives (Hyman, 1953).

There are at least two possible explanations of this phenomenon. The subject might be responding faster with a more probable response, or he might encode faster the more frequent stimulus. If a 1 to $1 \mathrm{~S}-\mathrm{R}$ mapping is used, these two possible interpretations cannot be separated.

In an ingenious study, LaBerge and Tweedy (1964) attempted to isolate stimulus probability from response probability. They used three stimuli (S1, S2, and S3) and two responses ( $\mathrm{Ra}$ and $\mathrm{Rb}$ ), such that $\mathrm{S} 1$ and $\mathrm{S} 2$ were associated with $\mathrm{Ra}$ and $\mathrm{S} 3$ was associated with $\mathrm{Rb}$. With this technique, variation in the relative frequency of the S1 and S2 should result in RT data that is analyzable with respect to the response vs. stimulus frequency interpretations of the relative frequency effect. Specifically, as LaBerge and Tweedy argue, if the relative frequency does not affect the stimulus processing stage, then the mean RTs for S1 and S2 should not differ. LaBerge and Tweedy used three colors as stimuli and two finger pressing responses and found that the more frequent of S1 and S2 was responded to faster. They concluded, therefore, that the speed of stimulus processing is affected by stimulus frequency.

There seems, however, to be a third possible explanations of the relative frequency effect. Response bias (the term used by LaBerge and Tweedy) could refer to two

Dean Purcell sponsors this paper and takes full editorial responsibility for its contents. distinct stages in the information processing system. One is the response selection stage, the other is the response execution stage. The LaBerge and Tweedy design allows variation in stimulus probability independently of response execution probability but not of the response selection probability. In other words, whereas $\mathrm{Ra}$ is a common response to both $\mathrm{S} 1$ and $\mathrm{S} 2$, there are two different response selections made by the subject, with different relative frequencies: S1-Ra and S2-Ra. It is conceivable that the more frequently the subject decides to respond with $\mathrm{Ra}$ to $\mathrm{S} 1$, the faster this decision will become, without necessarily affecting another decision of responding with $\mathrm{Ra}$ to $\mathrm{S} 2$. In the LaBerge and Tweedy technique, the frequency of the stimuli S1, S2, and S3 is perfectly confounded with the frequency of the decisions S1-Ra, S2-Ra, S3-Rb.

It seems impossible to solve this confounding directly. Indirectly, however, one can test whether the frequency of $\mathrm{Si}-\mathrm{Rj}$ decision rather than the frequency of Si processing is responsible for LaBerge and Tweedy's result. One can try to minimize the response selection time. If this leads to a decrease of the relative frequency affect in the LaBerge and Tweedy technique, then it must have been the frequency of the response decision that was responsible for their result.

The present experiment attempted to minimize response selection time by using highly compatible S-R mapping (Smith, 1968). This was achieved by using letter naming and using upper- and lowercase letters as the stimuli associated with the single response, the name of the letter. Thus A and a would appear with different frequencies, requiring naming them as a response. If the relative frequency of the stimulus affects its processing, the more frequent of the two should be responded to faster.

Hawkins, MacKay, Holley, Friedin, and Cohen (1973) performed a study very similar to the present one, essentially proposing the same hypothesis. Unfortunately the present authors were unaware of that study while conceiving of this one. The present study can serve 
as a successful replication of part of the Hawkins et al. one, with some modifications.

\section{METHOD}

Subjects

Eight undergraduate student volunteers from Oakland University served as subjects.

\section{Apparatus and Materials}

The stimulus materials consisted of cards with one of three color patches (gold, pink, or blue) or one of four letters (A, a, E, or e). The stimuli were presented to the subject via a tachistoscope. The onset of the stimulus triggered a clock which was stopped by the subject's vocal response into a microphone. RT was recorded to the nearest millisecond.

\section{Design and Procedure}

Each subject was tested in two sessions separated by approximately 1 to 2 weeks. The first session served as a replication of the LaBerge and Tweedy (1964) study. Subjects received a total of 340 trials with color patches as stimull, 100 practice, and 240 experimental trials. The pink card was the unique stimulus for all subjects, whereas gold and blue were both assigned to the same response. Four of the subjects had to respond vocally with " $A$ " to pink and "E" to gold and blue. The remaining four responded with "E" to pink and " $A$ " to gold and blue. The practice trials had pink appearing $40 \%$ of the time and gold and blue $30 \%$ each. In the first 120 of the 240 experimental trials, gold appeared with a probability of $50 \%$ and blue with a probability of $10 \%$ for half of the subjects. In the final 120 of the 240 experimental trials, the frequencies were reversed. For the remaining four subjects, the first frequent color was blue and the second was gold. Pink appeared with a probability of $40 \%$ throughout the experiment for all subjects. The sequencing of the stimuli was random within the following restrictions: (a) The same stimulus did not appear more than three times in a row. (b) The same response was not called for more than four times in a row. (c) Within each block of 20 trials, each stimulus appeared with a relative frequency corresponding to its probability for the experiment. Two randomized sequences were prepared and each was used for half of the subjects.

In the second session, the subjects' task was to name letters. Four of the subjects received A, E, and e as stimuli; the remaining four received $\mathrm{E}, \mathrm{A}$, and a (thus the unique stimulus was always uppercase). The relative frequency of the three possible letter stimuli, as well as their sequencing, was identical to that used in the color experiment. Each subject received the same randomizations in both sessions so that any sequencing effect would be identical for the high- and low-compatibility (letter and color stimuli, respectively) conditions.

Subjects were given standard RT instructions, stressing accuracy and speed within accuracy. They were informed if they made errors.

Subjects were instructed as to the statistical nature of the stimulus frequencies prior to the practice trials, prior to the first 120 experimental trials, and prior to the last 120 trials.

Each session lasted approximately $50 \mathrm{~min}$. Intertrial interval was about $5 \mathrm{sec}$. A verbal "ready" signal was given about $.5 \mathrm{sec}$ before stimulus presentation. Subjects were given a short break after the 100 practice trials and after the first 120 experimental trials.

\section{RESULTS AND DISCUSSION}

The mean RT for each subject for each of the four conditions (two relative frequencies and the two compatibility levels) was computed. Only correct responses were included in these means, and data from trials following an error were excluded. Overall error rate was low (less than 1\%), and errors correlated with RT. Table 1 shows the mean RTs collapsed across subjects for the unique stimulus, and for the two stimuli mapped onto the same response.

The critical test of the hypothesis proposed in this paper is the interaction of Compatibility by Relative Frequency of the two stimuli mapped onto the same response. If the hypothesis holds, then the relative frequency effect should be found for colors as stimuli more than for letters. A planned comparison for this interaction yielded a significant result $[t(7)=2.30, p<.05$, one-tailed], supporting the statement that response selection is the crucial factor in the LaBerge and Tweedy (1964) experiment.

As expected, the main effects for colors vs. letters and for frequency were both significant. Overall effect for type of stimulus (colors or letters) yielded $F(1,7)=$ $5.80, \mathrm{p}<.05$, letters being responded to faster than colors. A comparison for frequency of nonunique stimuli showed that frequent ones yield faster RT than nonfrequent ones $[t(7)=2.72, p<.05$, one-tailed] . A test for simple effects replicated the frequency effect for colors found by LaBerge and Tweedy $[\mathrm{t}(7)=2.65$, $\mathrm{p}<$ .05 , one-tailed] , but found insignificant frequency effect for letters $[t(7)=1.19 \mathrm{p}>.20]$. It should be noted, however, that frequent letters were responded to faster than infrequent ones, by $9 \mathrm{msec}$. It is possible that a more powerful experiment would have found it reliable. Thus, Miller and Pachella (1973) found a significant but small (about 17-msec) probability effect in a numeral naming task with eight alternatives. The present results suggest, however, that even if this effect is reliable, it is a very small one, and most of the relative frequency effect stems from the response selection stage.

The present results thus replicate the findings of Hawkins et al, (1973), who also found a relative frequency effect for low compatibility and failed to find it for high compatibility. However, one difference between the present results and those of Hawkins et al. should be noted: Hawkins et al. found the relative frequency effect on low-compatibility S-R pairing to disappear with practice. The results of the present experiment failed to find this effect, despite the fact that in the present experiment subjects received more trials (340 as compared to 240 in the Hawkins et al. experiment). In the present experiment, the advantage of frequent colors over infrequent colors was $60 \mathrm{msec}$ for the first 80 experimental trials and $71 \mathrm{msec}$ for the last 80 trials. The source of the discrepancy between the

Table 1

Mean Reaction Time in Milliseconds for Letters and Colors for Frequent, Infrequent, and Unique Stimuli

\begin{tabular}{lccc}
\hline & $\begin{array}{c}\text { Frequent } \\
(50 \%)\end{array}$ & $\begin{array}{c}\text { Infrequent } \\
(10 \%)\end{array}$ & $\begin{array}{c}\text { Unique } \\
(40 \%)\end{array}$ \\
\hline Colors & 477 & 541 & 508 \\
Letters & 444 & 453 & 445 \\
\hline
\end{tabular}


present results and those of Hawkins et al. is not clear to the present authors. It should be noted, though, that, even in the last block of trials in the Hawkins et al. experiment, there was a $32-\mathrm{msec}$ advantage to frequent stimuli over infrequent ones in the low-compatibility condition.

The conclusions presented here, as well as in the Hawkins et al. experiment, disagree with the conclusion of Miller and Pachella (1973). In two experiments (one using a character classification task, the other-a numerical naming task), Miller and Pachella manipulated the probability of the stimulus alternatives and their visual quality (by varying the contrast). They found a Probability by Contrast interaction, but not a Probability by Response Type interaction. Using the additive factor approach (Sternberg, 1969), they concluded that stimulus probability affects stimulus encoding and not response selection. However, Miller and Pachella (1973) found a much stronger probability effect in the character classification task than in the numeral naming task for both normal and degraded stimuli. In light of the present hypothesis, this result is readily interpretable: In a numeral naming task, the $\mathrm{S}-\mathrm{R}$ mapping is highly compatible; whereas in the character classification task, it is not. The Compatibility by Probability interaction, therefore, is evidenced in the
Miller and Pachella study. Their failure to find a Response Type (right vs. left hand) by Stimulus Probability interaction is not strong evidence against probability effect in the response selection stage, since there is no clear compatibility manipulation in that experiment.

\section{REFERENCES}

Hawkins, H. L., Mackay, S. L., Holley, S. L., Friedin, B. D., \& Cohen, S. L. Locus of the relative frequency effect in choice reaction time. Journal of Experimental Psychology, 1973, 101, 90.99.

Hick. W. E. On the rate of gain of information. Quarterly Journal of Experimental Psychology, 1952, 4, 11-26.

Hyman, R. Stimulus information as a determinant of reaction time. Journal of Experimental Psychology, 1953, 45, 188-196.

LABerge, D. \& Tweedy, J. R. Presentation probability and choice time. Journal of Experimental Psychology, 1964 , 68, 477-481.

Miller, J. O., \& Pachella, R. G. Locus of the stimulus probability effect. Journal of Experimental Psychology, 1973, 101, 227.231.

Smith, E. E. Choice reaction time: An analysis of the major theoretical positions. Psychological Bulletin, 1968, 69, 77-110.

StERnBERG, S. The discovery of processing stages: Extensions of Donder's method. In W. G. Koster (Ed.), Attention and performance II. Acta Psychologica, 1969, 30, 276-315.

(Received for publication February 19, 1976.) 